American Journal of Biochemistry and Biotechnology 1 (2): 69-73, 2005

ISSN 1553-3468

(C) Science Publications, 2005

\title{
Expression, Purification and Activity Assay of Two New Recombinant Antagonists of Fibrinogen Receptor
}

\author{
Jianbo Yang, Jia Yao, Kun Yang, Zichun Hua and Jie Yang \\ State Key Laboratory of Pharmaceutical Biotechnology \\ Nanjing University, Nanjing 210093, China
}

\begin{abstract}
The gene sequence of Decorsin which is extracted from a kind of North American leeches was synthesized. Two recombinant proteins, Annexin V plus Decorsin (AnnV-D39) and Annexin V plus the carboxyl terminal 27 amino acid residues of Decorsin(AnnV-D27), were constructed. And a 10 amino acids linker peptide of GGGGSGGGGS was inserted between Annexin V and Decorsin in AnnV-D39. Using pET-28(a+) as an expressing vector, both two recombinant proteins were expressed in E. Coli BL21(DE3) with high efficiency as inclusion bodies. The expression products were purified by DEAE-Cellulose 52 and Sepharose CL-4B chromatography under denaturing condition. Platelet Aggregation Assay (PAA) shows that AnnV-D39 has good anti-platelet aggregation activity. However, AnnV-D27 shows no such activities in any PAA test. AnnV-D39 shows good anti-platelet aggregation activity as a new antagonist of fibrinogen receptor, while Annv-D27 needs re-modification.
\end{abstract}

Key words: Antagonist of fibrinogen receptor, anti-platelet aggregation activity, annv-Decorsin fusion

\section{INTRODUCTION}

Many diseases, such as coronary artery disease, coronary heart disease and stroke, have proven to be closely related with thrombosis. These diseases cause about 15 million death cases per year in the world and badly hurt the health of human beings ${ }^{[1]}$. Up to now, many anti-thrombus drugs have been developed, in which anti-platelet agents show great promise ${ }^{[2]}$. Decorsin, a protein isolated from the North American leech Macrobdella decora, acts as an antagonist of platelet glycoprotein IIb-IIIa (GPIIb-IIIa)------the fibrinogen receptor and is a potent inhibitor of platelet aggregation. The primary sequence of decorsin indicates that the protein is 39 amino acids long and contains 6 cysteine and 6 proline residues, as well as the sequence Arg-Gly-Asp, (RGD), a proposed recognition site of many adhesion proteins ${ }^{[3]}$. Annexin $\mathrm{V}$, belonging to a family of proteins which was first discovered in 1990, the annexins, with anticoagulant properties has proven to preferentially bind to negatively charged phospholipids like PS (phosphatidylserine), which is greatly exposed on their membranes when platelets are activated, in the presence of $\mathrm{Ca}^{2+[4-6]}$.

Though pharmacological studies have demonstrated that Decorsin might be well suited for the prophylaxis and therapy of various thrombotic diseases as an anti-platelet drug, the limited availability of natural Decorsin precludes its application in clinical testing and therapy. To solve the problem, production of recombinant Decorsin in large quantities by DNA, recombinant technology offers an effective means.

With the help of Computer-Assistant-Design (CAD), we use homologous molecular modeling to predict the active site of Decorsin, a polypeptide including the whole 27 carboxyl-terminal amino acid residues, which includes the recognizing and binding site of human platelet glycoprotein IIb-IIIa (GPIIb-IIIa)------the Arg-Gly-Asp(RGD) sequence ${ }^{[7-9]}$. Thus, we design and construct two recombinant proteins------AnnV-D39, the recombinant of Annexin V and the whole 39 amino acid residues of Decorsin and Annv-D27, the recombinant of Annexin V together with the 27 carboxyl-terminal amino acid residues. Then, we express the two recombinant proteins in the bacteria Escherichia coli (E.coli), which is an important and most frequently used host organism in the production of recombinant proteins, in order to achieve the efficient expression of new and pure active antagonists of platelet glycoprotein IIb-IIIa (GPIIb-IIIa) after a series of purification.

\section{MATERIALS AND METHODS}

Kits and main reagents: Restriction enzymes Nco I, BamH I, Sal I, Hind III and T4 ligase were purchased from MBI Fermentas Company (Canada). Pfu-taq DNA polymerase and Uniq-10 DNA Retraction Kit were from Shanghai Sagon Biotech Company (China).

Corresponding Author: Yang Jie, State Key Laboratory of Pharmaceutical Biotechnology, School of Life Sciences, Nanjing University, 22 HanKou Road, Nanjing, 210093, PR. China;

Tel: 862583594060 Fax: 86-25-83324605 
Bradford Reagents, ADP were from Amresco (USA). Sepharose Cl-4B gel and DEAE Cellulose 52 resin were from Pharmacia Company. Vivaspin 50 ultrafiltration tube was from Satorius Company. Thrombin from human plasma was purchased from Sigma Company. To measure the aggregation of platelet we used CHRONO-LOG490-4D platelet aggregation analysis machine.

Vector and host strain: E.coli strain, BL21 (DE3), was supplied by our own lab and expression vector, pET28a $(+)$ was purchased from Invitrogen Company. Plasmid, pET19a(+)-AnnV was provided by Dr. Hua Zichun in State Key Laboratory of Pharmaceutical Biotechnology of Nanjing University, which includes the cDNA sequence of Annexin V. Plasmid pYJD-Decorsin containing the cDNA sequence of Decorsin was synthesized by Shanghai Bocai Biotech Company (China).

Primer synthesis and DNA sequencing: DNA Primer was synthesized by Shanghai Sangon Company (China) and DNA Sequencing was accomplished also by Shanghai Sangon Company (China).

Construction of pET28a(+)-AnnV-D39 and pET28a(+)-AnnV-D27 expression vectors: Annexin $\mathrm{V}$ cDNA sequence was obtained by the following PCR procedure with plasmid pET19a(+)-AnnV as template: (1) $94^{\circ} \mathrm{C}$ for $3 \mathrm{~min}$; (2) 30 cycles at $94^{\circ} \mathrm{C}$ for $30 \mathrm{sec}, 55^{\circ} \mathrm{C}$ for $45 \mathrm{sec}, 72^{\circ} \mathrm{C}$ for $90 \mathrm{sec}$; (3) $72^{\circ} \mathrm{C}$ for $10 \mathrm{~min}$. The two primers were Primer 15'CgCggATCCATggCACAggTTCTCAg-3' and Primer 2 5'-CgggATCCgTCgACCATCTTCTC- CACA -3'. The whole sequence of Decorsin (D39) together with its N-terminal linker peptide GGGGSGGGGS DNA sequence was obtained by the following PCR procedure with plasmid pYJD-Decorsin as template: (1) $94^{\circ} \mathrm{C}$ for $3 \mathrm{~min}$; (2) 30 cycles at $94^{\circ} \mathrm{C}$ for $30 \mathrm{sec}, 59^{\circ} \mathrm{C}$ for $45 \mathrm{sec}$, $72^{\circ} \mathrm{C}$ for $45 \mathrm{sec}$; (3) $72^{\circ} \mathrm{C}$ for $10 \mathrm{~min}$. The two primers for this procedure were Primer 3 5'CTgCTgCTgCCACCgTAATACgACTCAC-3' and Primer 4 5'-gACTCgAgggATCCAgATCTCCAgTCTTC-3'. The DNA sequence of Decorsin's Cterminal 27 amino-peptide (D27) was obtained in the following PCR procedure also with pYJD-Decorsin as template: (1) $94^{\circ} \mathrm{C}$ for $3 \mathrm{~min}$; (2) 30 cycles at $94^{\circ} \mathrm{C}$ for $30 \mathrm{sec}, 56^{\circ} \mathrm{C}$ for $45 \mathrm{sec}, 72^{\circ} \mathrm{C}$ for $45 \mathrm{sec}$; (3) $72^{\circ} \mathrm{C}$ for $10 \mathrm{~min}$. The primers of this reaction were Primer4 5'gACTCgAgggATCCAgATCTCCAgTCTTC-3' and Primer5 5'-gCgTCgA- CCCATggAAAAATgCCTgTg3'. All PCR products are purified by agarose electrophoresis and gel retraction.

AnnV sequence was inserted into plasmid $\mathrm{pET} 28 \mathrm{a}(+)$ at the site of Nco I and BamH I to get intermediary plasmid pET28a(+)-AnnV. Then D39 and D27 were respectively inserted into pET28a(+)-AnnV plasmid at the site of Sal I and Hind III in order to get the correspondent expression vectors pET28a(+)-AnnVD39 and pET28a(+)-AnnV-D27. Both two expression vectors were transformed into E.coli $\mathrm{DH} \alpha 5$ in order to verify the correct sequence through sequence analysis and get enough quantity for the next step under the directions of certain references ${ }^{[10,11]}$. (Fig. 1 and 2)

Induction and expression of the recombinant proteins AnnV-D39 and AnnV-D27: The obtained expression vectors were transformed into BL21 (DE3) fertilized on LB ( $30 \mu \mathrm{g} / \mathrm{ml}$ Kanamycin included) plate at $37^{\circ} \mathrm{C}$ overnight. Single clone was selected to be cultured in LB liquid overnight. And inoculate $1 \%$ volume of culture into the fresh LB medium. IPTG was added (at a final concentration of $1 \mathrm{mmol} / \mathrm{L}$ ) when O.D. 600 of the suspension is up to 0.6. Cells were precipitated after $3 \mathrm{~h}$ subsequently culturing and resuspended in PBS. Twenty-five microliter of the suspension was taken into $25 \mu \mathrm{l}$ SDS-PAGE sample buffer $(2 \mathrm{X})$ and heated for $5 \mathrm{~min}$ at $97^{\circ} \mathrm{C}$. Each sample of $2 \mu \mathrm{l}$ was added on $15 \%$ SDS-PAGE tricine gel. The level of expression was determined using SDS-PAGE followed by densitometor scanning (Fig. 4).

Cell lysis and inclusion body solubilization: Cells from $1 \mathrm{~L}$ of fermentated suspension were harvested by centrifugation, resuspended in $20 \mathrm{ml}$ lysis buffer (50mmol/L Tris.HCl, $1 \mathrm{mmol} / \mathrm{L}$ EDTA, $1 \mathrm{mmol} / \mathrm{L}$ DTT, $5 \%$ glycerol, 50mmol/L glucose, $1 \mathrm{mmol} / \mathrm{L}$ PMSF, $5 \mathrm{mg} / \mathrm{ml}$ Leupeptin, 10mg/ml Aprotinin, PH8.0) broken down with ultrasonic waves and centrifuged. The recombinant proteins were in the precipitate as inclusion bodies. The inclusion bodies were washed in $20 \mathrm{ml}$ lysis buffer without $1 \%$ Triton-100 twice. Washed inclusion bodies were centrifuged to move excess liquid and resuspended in $20 \mathrm{ml}$ denaturing buffer $(50 \mathrm{mmol} / \mathrm{L}$ Tris.HCl, 10mmol/L EDTA, 10mmol/L EDTA, 10mmol/L DTT, 8mol/L Urea, 1mmol/L PMSF, 5mg/ml Leupeptin, 10mg/ml Aprotinin, PH8.0), mixed round by magnetic stirring apparatus at room temprature for 4 hours, then centrifugated for $15 \mathrm{~min}$ at $4^{\circ} \mathrm{C}$ at $32000 \mathrm{~g}$. The supernatant was collected.

Purification of recombinant proteins under denaturing conditions: The supernatant mixture was firstly fed into the prepared DEAE Cellulose 52 column. The Column was washed and equilibrated with Buffer A (8mol/L Urea, 50mmol/L Tris. $\mathrm{HCl})$. The mixture was eluted with linear gradient from Buffer $\mathrm{A}$ $(8 \mathrm{~mol} / \mathrm{L}$ Urea, $50 \mathrm{mmol} / \mathrm{L}$ Tris. $\mathrm{HCl})$ to Buffer B (8mol/L Urea, 50mmol/L Tris. $\mathrm{HCl}, 150 \mathrm{mmol} / \mathrm{L} \mathrm{NaCl})$. 


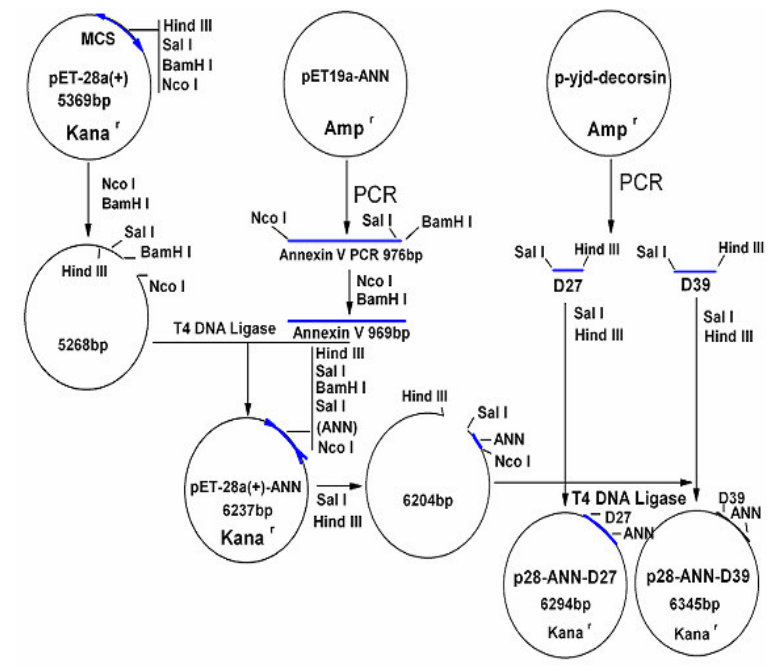

Fig. 1: The process of constructing expression vectors: pET28a(+)-AnnV-D39 and pET28a(+)AnnV-D27

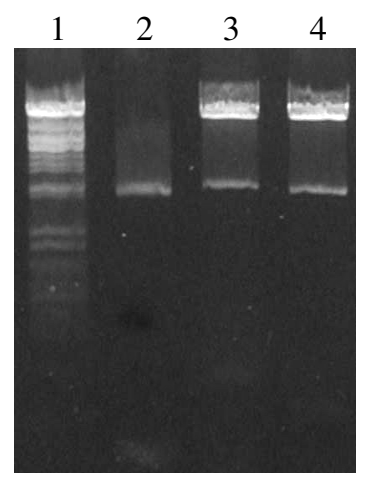

Fig. 2: Analysis of pET28a (+)-AnnV-D39 and pET28a (+)-AnnV-D27 by restriction enzymatic digestion Lane 1: Marker: Lamda DNA/Exo471 M.W. Lane 2: PCR product of AnnexinV ( 980 bp). Lane 3: pET28a (+)- AnnVD39/NcoI+Hind III (AnnV-D39: 1131bp). Lane 4: pET28a(+)-AnnV-D27/Nco I+Hind III (AnnV-D27: 1056bp)

SDS-PAGE gels were used to analyze elution fractions corresponding to $\mathrm{UV}$ absorbance $\left(\mathrm{OD}_{280} \mathrm{~nm}\right)$ chromatogram peaks. The achieved active components were precipitated by double volume ice-chilled ethanol, dissolved in $4 \mathrm{ml}$ denaturing buffer then fed into prepared Sepharose CL-4B column and eluted with the buffer $(8 \mathrm{~mol} / \mathrm{L}$ Urea, $50 \mathrm{mmol} / \mathrm{L}$ Tris. $\mathrm{HCl}, 150 \mathrm{mmol} / \mathrm{L}$ $\mathrm{NaCl}$ ). Also the elution fractions corresponding to UV absorbance $\left(\mathrm{OD}_{280} \mathrm{~nm}\right)$ chromatogram peaks were collected and analyzed by SDS-PAGE gels. (Fig. 4)

Renaturation of the recombinant proteins: The achieved active elution fractions were precipitated by double volume ice-chilled ethanol then dissolved in $2 \mathrm{ml}$ denaturing buffer. Add the denatured proteins into $200 \mathrm{ml}$ renaturing buffer $(100 \mathrm{mmol} / \mathrm{L}$ Tris. $\mathrm{HCl}$, $2 \mathrm{mmol} / \mathrm{L}$ EDTA, $1 \mathrm{~mol} / \mathrm{L}$ arginine, $5 \mathrm{mmol} / \mathrm{L}$ reduced glutathione, $0.5 \mathrm{mmol} / \mathrm{L}$ glutathione, $\mathrm{PH} 7.4$ ) at $4{ }^{\circ} \mathrm{C}$, mixed by magnetic stirring apparatus. Repeat the above procedures four times at an interval of 8 hours each. The mixture stood renaturing for 36 hours, dialyzed by dialysis buffer $(50 \mathrm{mmol} / \mathrm{L}$ Tris. $\mathrm{HCl}, 100 \mathrm{mmol} / \mathrm{L} \mathrm{NaCl}$, $\mathrm{PH} 7.4$ ) at $4^{\circ} \mathrm{C}$ for 72 hours and finally concentrated by freeze-dry method. Buffer should be changed by ultrafiltration before using.

Measuring the anti-platelet-aggregation activity of the recombinant proteins: The antiplatelet aggregation activity was determined by Born Turbidimetry. Blood were from healthy adults who had been excluded from any drugs with anti-platelet aggregation activities for 10 days. Human Platelet-Rich Plasma (PRP) and Human Platelet-Poor Plasma (PPP) were prepared for the assays. First $300 \mu \mathrm{PRP}$ plus $35 \mu \mathrm{l}$ sample of different concentrations in $0.9 \% \mathrm{NaCl}$ was incubated in an aggregometer at $37^{\circ} \mathrm{C}$ for $1 \mathrm{~min}$. A total of $15 \mu \mathrm{lDP}$ ( at a final concentration of $10 \mu \mathrm{mol} / \mathrm{L}$ ) was added and the change of light transmission was recorded over $5 \mathrm{~min}$. Transmission was set at $100 \%$ for PPP and $0 \%$ for PRP. For the assays using thrombin as an agonist of platelet aggregation, followed the above procedure except for using $15 \mu$ l thrombin (at a final concentration of $1 \mathrm{U} / \mathrm{ml}$ ) instead of ADP.

\section{RESULTS}

Construction of Expression Vectors: As indicated by agarose electrophoresis, the obtained AnnV-D39 and AnnV-D27 were about 1131 bps and 1056 bps respectively. (Lane 3 and Lane 4 in Fig. 2) DNA sequencing analysis confirmed that the DNA sequence and protein sequence were correct (Fig. 3).

Induction and Purification of the recombinant proteins: Based on SDS-PAGE assay followed by densitometry scanning, the amount of the recombinant proteins reached $60 \%$ of the total proteins in inclusion bodies. After purification the purity of the obtained denatured recombinant protein was confirmed as high as $95 \%$ by SDS-PAGE. The molecular mass of recombinant proteins AnnV-D39 and AnnV-D27 were $41 \mathrm{kD}$ and $39 \mathrm{kD}$, respectively. (Fig. 4)

Anti-platelet aggregation activities of recombinant proteins: Using $10 \mu \mathrm{mol} / \mathrm{L}$ ADP and $1 \mathrm{U} / \mathrm{mL}$ thrombin as aggregation agonists respectively. The concentration of proteins were determined by BCA method and $0.9 \%$ $\mathrm{NaCl}$ was taken as Negative Control (NC). The Maximum Aggregation Rate(MAR) of platelet in $5 \mathrm{~min}$ 
Am. J. Biochem. and Biotech., 1 (2):69-73, 2005

Table 1: Inhibition of ADP and Thrombin-induced platelet aggregation in human PRP

\begin{tabular}{|c|c|c|c|c|c|}
\hline Group & Concentration $\square \mu \mathrm{mol} / \mathrm{L} \square$ & $\mathrm{MAR}_{\mathrm{ADP}} \% \square$ & $\mathrm{AIR}_{\mathrm{ADP}} \% \square$ & $\mathrm{MAR}_{\mathrm{THR}} \% \square$ & $\mathrm{AIR}_{\mathrm{THR}} \% \square$ \\
\hline$\overline{\mathrm{NC}}$ & NA & $58.8 \pm 4.1$ & NA & $77.3 \pm 5.8$ & NA \\
\hline A1 & 2.573 & $18.6 \pm 2.7$ & 68.4 & $3.7 \pm 2.2$ & 95.2 \\
\hline A2 & 1.801 & $21.7 \pm 3.8$ & 63.1 & $5.1 \pm 2.6$ & 93.4 \\
\hline A3 & 0.772 & $25.6 \pm 2.6$ & 56.5 & $10.1 \pm 4.8$ & 86.9 \\
\hline A4 & $2.573 \times 10^{-1}$ & $32.3 \pm 4.8$ & 45.1 & $26.6 \pm 5.9$ & 65.6 \\
\hline A5 & $2.573 \times 10^{-2}$ & $40.1 \pm 6.0$ & 31.8 & $38.3 \pm 6.1$ & 50.5 \\
\hline A6 & $2.573 \times 10^{-3}$ & $47.2 \pm 3.9$ & 19.7 & $57.0 \pm 5.8$ & 26.3 \\
\hline A7 & $2.573 \times 10^{-4}$ & $52.0 \pm 2.7$ & 11.6 & $63.2 \pm 7.9$ & 18.2 \\
\hline B1 & 2.172 & $65.4 \pm 5.2$ & 0 & $76.9 \pm 8.7$ & 0 \\
\hline $\mathrm{IC}_{50}(\mathrm{ADP})$ & \multirow{2}{*}{\multicolumn{5}{|c|}{$\begin{array}{l}300.8 \mathrm{nmol} / \mathrm{L} \\
14.4 \mathrm{nmol} / \mathrm{L}\end{array}$}} \\
\hline $\mathrm{IC}_{50}$ (THR) & & & & & \\
\hline
\end{tabular}

\section{1: AnnexinV-GGGGSGGGGSAMAPAPRLPQCQGDDQEKCLCNKDECPPGQCRFPRGDADPYCE}

2: AnnexinV-_-_-_-_-_-_-_-_-_-EKCLCNKDECPPGQCRFPRGDADPYCE

Fig. 3: The amino acid sequences of AnnV-D39 and AnnV-D27 respectively

1) amino acid sequence of AnnV-D39 containing the full length of both Annexin V and Decorsin with a linker peptide GGGGSGGGGS. 2) amino acid sequence of AnnV-D27 containing AnnexinV and the 27 amino acids peptide variant of Decorsin without a link peptide

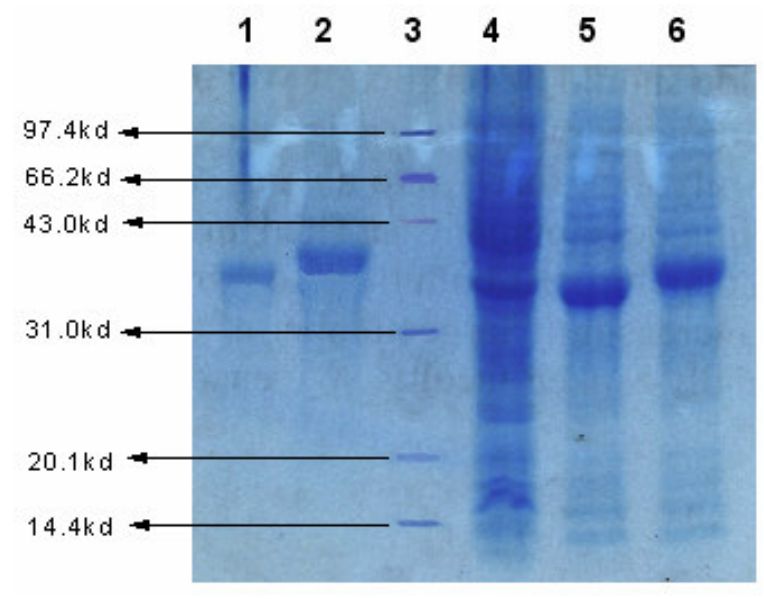

Fig. 4: SDS-PAGE analysis of recombinant protein AnnV-D39 and AnnV-D27

Lane 1: purified recombinant protein AnnV-D27 after two-steps purification

Lane 2: purified recombinant protein AnnV-D39 after two-steps purification

Lane 3: protein M.W. marker ranging from 97.4, $66.2,43.0,31.0,20.1$ to $14.4 \mathrm{kD}$. (from top to bottom)

Lane 4: total protein expressed by E.coli BL21 $(\mathrm{DE} 3) / \mathrm{pET} 28 \mathrm{a}(+)$, induced by $1 \mathrm{mmol} / \mathrm{L}$ IPTG.

Lane 5: total protein from washed inclusion body expressed by E.coli BL21 (DE3)/pET28a (+)-AnnV-D27, induced by $1 \mathrm{mmol} / \mathrm{L}$ IPTG

Lane 6: total protein from washed inclusion body expressed by E.coli BL21 (DE3)/pET28a (+)-AnnV-D39, induced by $1 \mathrm{mmol} / \mathrm{L}$ IPTG

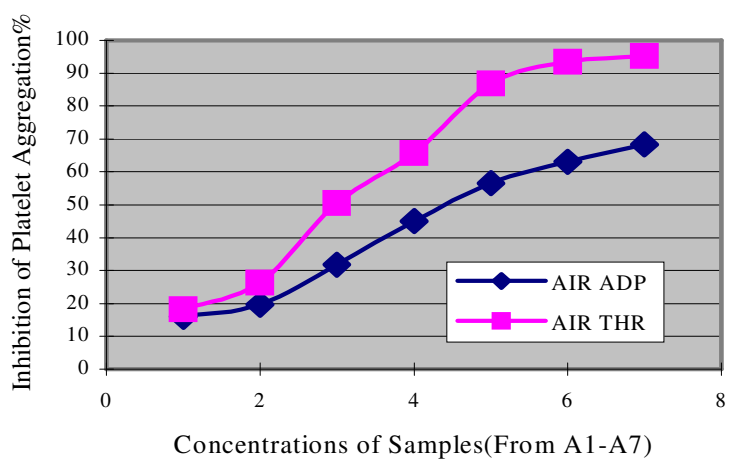

Fig. 5: Inhibition of ADP and thrombin induced aggregation

was recorded. Samples' Aggregation Inhibition Rate (AIR) was calculated in the formula: $\mathrm{AIR}_{\text {sample }}=\left(1-\mathrm{MAR}_{\text {sample }} / \mathrm{MAR}_{\mathrm{NC}}\right)$. Samples' $\mathrm{IC}_{50}$ were calculated by Berkson-Logit method.(Table 1)

Annv-D39 showed great inhibition activity to platelet aggregation induced by thrombin. AnnV-D39 at a concentration of $2.573 \mu \mathrm{mol} / \mathrm{L}$ showed a more than $84 \%$ inhibition rate to platelet aggregation induced by thrombin above $15 \mathrm{U} / \mathrm{ml} \quad\left(\mathrm{MAR}_{\mathrm{NC}}=98.6, \mathrm{MAR}_{\mathrm{AnnV}}\right.$ ${ }_{\text {DD39 }}=18.3 \pm 7.4$ )(Fig. 5). However, AnnV-D27 did not show any obvious inhibition activity to platelet aggregation induced either by ADP or by thrombin. $\mathrm{X}$-Value different concentrations of Samples as characterized in Table 1.

\section{DISCUSSION}

In this study, we designed two different recombinant proteins, AnnV-D39 and AnnV-D27. 
AnnV-D39 showed good anti-platelet aggregation activity. As demonstrated in the study, IC $_{50}$ AnnV-D39's inhibition activity to ADP induced platelet aggregation is about $308 \mathrm{nmol} / \mathrm{L}$, which is slightly lower than that of natural Decorsin (about 500nmol/L) ${ }^{[3]}$. In the case of thrombin induced platelet aggregation, AnnV-D39 showed great inhibition activity, in which the corresponding $\mathrm{IC}_{50}$ reached $14.4 \mathrm{nmol} / \mathrm{L}$. Such phenomena were consistent with the results confirmed in others' study. Former studies show that Annexin V doesn't bind specifically with activated platelet induced by ADP, which doesn't cause PS, the receptor of Annexin V, to expose on the outer membrane ${ }^{[11]}$. But in the case of thrombin induced platelet activation accompanied by the great exposure of PS on the membrane of platelet, Annexin V binds with PS and causes the increase of Decorsin's local concentration around platelets, which will consequently enhance Decorsin's anti-platelet aggregation activity greatly as was demonstrated in our study. And the fact that AnnV-D27 didn't have any anti-aggregation activity no matter whether platelets were induced by ADP or thrombin helps to show that despite of its anti-coagulant properties which has been confirmed in former studies, Annexin V alone does not show much of antiaggregation activity, however, it in some sense help to concentrate Decorsin around platelets with PS greatly exposed on the membrane. And in order to get further confirmation, more experiments are necessary, such as analysis of changes in AnnV-D39's anti-platelet aggregation activities along with other different inducers. And also in vivo experiments are to be taken.

As for the incompetence of AnnV-D27 in the inhibition of platelet aggregation, we think it is due to the following reasons: 1) AnnV-D27 doesn't have a linker peptide, which may cause the smaller D27 sequence unable to expose its active site in the recombinant protein because of the steric hindrance between protein subunits and inhibit its antiaggregation activity. 2) The $12^{\text {th }}$ amino acid in Decorsin's N-terminal sequence contributes to the maintenance of active site indirectly or it is important to the recognition between of GPIIb/IIIa and RGD sequence.

Taken together, we successfully constructed and expressed the recombinant protein AnnV-D39 with good inhibition activity to platelet aggregation especially in those induced by thrombin. And AnnV-D27 needs re-modification to achieve wanted activity.

\section{ACKNOWLEDGEMENTS}

This work was financially supported by the Natural Science Foundation of China (No.30171094 and 30271497), also was supported by Foundation of backbone teachers of Education Ministry of China.

\section{REFERENCES}

1. Murray, C.J., A.D. Lopez, 1996. The Global Burden of Disease: A Comprehensive Assessment of Mortality and Disability from Diseases, Injuries and Risk Factors in 1990 and Projected to 2020. Cambridge: Harvard University Press; 17: 176-178.

2. Patrono, C., F. Bachmann, C. Baigent, C. Bode, R. De Caterina, B. Charbonnier et al., 2004. Expert Consensus Document on the Use of Antiplatelet Agents. The Task Force on the Use of Antiplatelet Agents in Patients with Atherosclerotic Cardiovascular Disease of the European Society of Cardiology. Eur. Heart J., 252: 166-181.

3. Seymour, J.L., W.J. Henzel, B. Nevins, J.T. Stults and R.A. Lazarus, 1990. Decorsin. A potent glycoprotein IIb-IIIa antagonist and platelet aggregation inhibitor from the leech Macrobdella decora. J. Biol. Chem., 265: 10143-10147.

4. Tait, J.F. and D. Gibson, 1992. Phospholipid binding of Annexin V: Effects of calcium and membrane phosphotidylserine content. Arch Biochem. Biophy., 298: 187-191

5. Gerke, V. and S.E. Moss, 1997. Annexins and membrane dynamics, Biochimica et Biophysica Acta, 1357: 129-154

6. Bevers, E.M., P. Comfurius and R.F. Zwaal, 1983. Changes in membrane phospholipid distribution during platelet activation. Biochim Biophys Acta, 736: 57-66.

7. Krezel, A.M., J.S. Ulmer, G. Wagner and R.A. Lazarus, 2000. Recombinant decorsin: dynamics of the RGD recognition site. Protein Sci., 9: 1428-1438.

8. Lapchak, P.A. ande D.M. Araujo, 1996. Therapeutic Potential of Platelet Glycoprotein IIb/IIIa Receptor Antagonists in the Management of Ischemic Stroke. J. Biol. Chem., 271: 17785-17790

9. Padoin, E., A. Alexandre, L. Cavallini, P. Polverino de Laureto, G.H. Rao and M.G. Doni, 1996. Human Platelet Activation Is Inhibited by the Occupancy of Glycoprotein IIb/IIIa Receptor. Arch Biochem. Biophys., 333:407-413.

10. Joe Sambrook, David Russell, 2001. Molecular Cloning: A Laboratory Manual, $3^{\text {rd }}$ Ed. Cold Spring Harbor Lab (CSHL) Press.

11. Perumal, T. and F.T. Jonathan, 1990. Binding of Annexin V/Placental Anticoagulant Protein I to Platelets : Evidence for Phosphotidylserine Exposure in the Procoagulant Response of Activated Platelets. The J. Biolog. Chem., 29: 17420-17423. 